\title{
Applications of Spectral Analysis Techniques for Estimating the Nordic Grid's Low Frequency Electromechanical Oscillations
}

\author{
Luigi Vanfretti* Sebastian Bengtsson* \\ Vemund H. Aarstrand ${ }^{* *}$ Jan O. Gjerde** \\ * KTH Royal Institute of Technology, Stockholm, Sweden \\ ** Statnett SF, Research \& Development, Oslo, Norway
}

\begin{abstract}
For power networks such as the Nordic Grid, that have operation constraints limits imposed by the existence of low-damped electromechanical oscillations, the estimation of electromechanical mode properties is of crucial importance for providing power system control room operators with adequate indicators of the stress of their network. This article addresses the practical application of different spectral analysis techniques that can be used for the estimation of electromechanical mode properties using data emerging from real synchronized phasor measurement units (PMUs) located at both the low-voltage distribution and high-voltage transmission networks of the Nordic grid. Emphasis is made on providing systematic approaches to deal with imperfect data found in practice so that accurate estimates can be computed.
\end{abstract}

\section{INTRODUCTION}

With the continuous increase in the demand for reliable electricity supply coupled by the on-going trend for more interconnections across different power networks and the increase of variable generation sources, the dynamics of power grids are becoming increasingly complex. This is true, particularly for those power networks such as the Nordic grid [1], that have operation constraints due to limits imposed by low-damped electromechanical oscillations. Hence, the estimation of electromechanical mode properties [2], that is the frequency and damping of electromechanical oscillations and their associated mode shapes, is of crucial importance for providing power system control room operators with adequate indicators of the stress of their network $[3]^{1}$.

This article describes how spectral analysis techniques can be used for the estimation of mode properties using data emerging from real synchronized phasor measurent units (PMUs) located at both the low-voltage distribution and high-voltage transmission networks of the Nordic Grid. The present study extends previous work using data from the Nordic grid [4], by addressing the challenges brought by actual data imperfections found in field measurements. Recognizing data imperfections, undesirable spectral components in the data sets, and attacking them through the design and tuning of proper algorithms may allow for a more rapid integration of these techniques within tools used at control centers [2] in the Nordic grid.

\footnotetext{
* Invited Paper - Special Session: "Development of System ID Methods for Power System Dynamics", 16th IFAC Symposium on System ID (SYSID), 2012.

Luigi Vanfretti is supported by the STandUP for Energy collaboration initiative and the KTH School of Electrical Engineering.

Sebastian Bengtsson was supported in part by the EIT InnoEnergy Collocation Center Sweden within the "Smart Power" Thematic Area.

1 Due to space limits, only key references are provided in this article. Note that [3] and [2] are comprehensive book chapters providing a large amount of
} references.
The remainder of this article is organized as follows. Section 2 proposes different pre-processing techniques for conditioning data from the Nordic grid, a sound rationale is given for the application of each step in this process. In Section 3 parametric and nonparametric spectral estimation techniques are applied to ambient PMU data, and parameter selection is discussed. Section 4 gives a comparison of the different estimators and, more importantly, provides evidence of the existence of forced oscillations in measurements from both the low-voltage and highvoltage networks. For mode damping estimation, Section 5 presents three algorithms and an evaluation of their performance using simulated data; the effect of forced oscillations on these algorithms is explained. Finally, in Section 6, we outline the main contributions of this article and outstanding challenges for mode property estimation.

\section{PREPROCESSING}

It is not uncommon for PMU data to suffer from data quality issues. Before using phasor measurement data for mode estimation purposes, the data must be preprocessed to remove flawed and redundant data and, when possible, to add estimates of missing samples. Moreover, spectral analysis techniques require data from a stochastic process $[2,3]$ to be able to work properly. To this aim, deterministic components in the signals must be removed.

\subsection{Removal of bad data}

Bad data is common and exists in many forms; the most frequently occurring types are related to internal PMU failures to update or retrieve sample information. This will results in the following forms of bad data: $(i)$ sequences of identical timestamps and/or values, and (ii) samples with value zero. Errors such as these are easily addressed by detecting and removing imperfect data.

2.2 Removal of outliers

Outliers will cause the performance of filters to deteriorate, this will in turn result in poor spectral estimates. Consequently, outliers must be removed before data is 


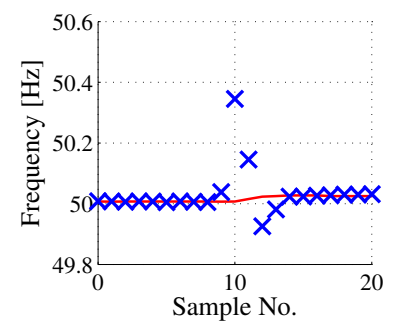

(a)

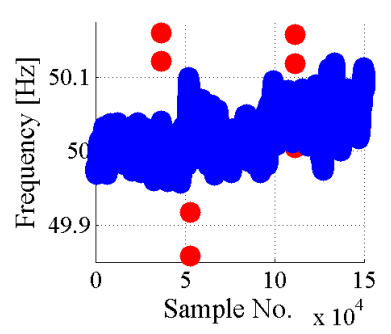

(b)
Figure 1. (a) Data with five outliers, moving median (red line) and measurements (blue crosses), (b) Data from Hasle showing removed outliers (red dots).

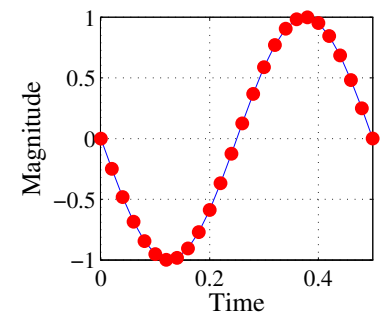

(a)

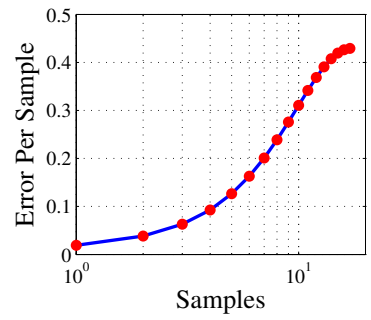

(b)
Figure 2. (a) $2 \mathrm{~Hz}$ oscillation, $50 \mathrm{~Hz}$ sampling rate(b) Error per interpolated samples for a $2 \mathrm{~Hz}$ oscillation depending on the number of adjacent missing samples.

filtered. Data comprised by a large amount of oscillations and with moving mean set very high requirements on the the algorithm that detects outliers. Methods commonly used for detection of outliers in stationary time series will yield unsatisfactory results when applied on this sort of data. An algorithm using the moving median of the signal, capable of taking into account the moving mean to detect outliers, is suitable for this task. Results from applying such algorithm are shown in Fig.1(a). The algorithm computes the moving median (using a median filter of order 10) of the signal and removes samples that deviate more than $k$ standard deviations ${ }^{2}$ from the moving median. Fig.1(b) shows the outliers detected with the the moving median algorithm.

\subsection{Interpolation}

Estimates replacing missing samples, as well as removed data and outliers, are computed using interpolation. Because electromechanical modes can be observed in the frequency region up to $2 \mathrm{~Hz}$ [3] the fastest dynamics we need to take into consideration is a $2 \mathrm{~Hz}$ oscillation, in Fig.2(a) such an oscillation is depicted. For the sampling rate of $50 \mathrm{~Hz}$, there are 25 samples distributed over one period of the oscillation. As long as there is not more than a few samples missing, linear interpolation will give good estimates. But in cases when there are larger blocks of missing data, interpolation will not yield good estimates and therefore should be avoided, as depicted in Fig. 2(b).

\subsection{Data parceling}

Filters require data without gaps due to missing data in order to be able to perform properly. To this end the data is split into parcels without any missing samples, and then the individual parcels are processed separately.

2 Note that since the data characteristics from different units and
locations can vary slightly it is therefore important that an appro-
priate value of $k$ is selected for each PMU for optimal performance.

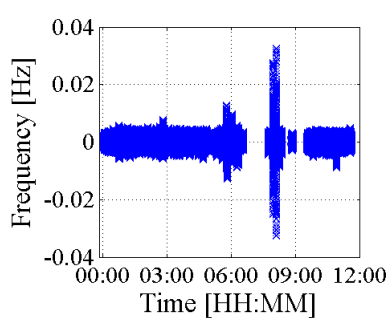

(a)

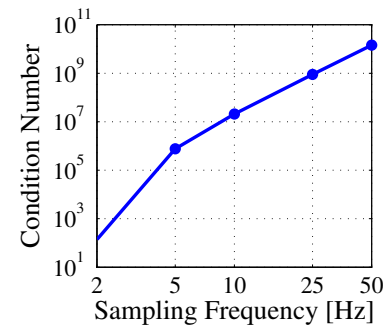

(b)

Figure 3. (a) Data from Hasle after extracting the signals mean and low pass filtering, (b) Condition number of the Yule-Walker autocovariance matrix for different sampling frequencies.

\subsection{Mean removal and high pass filtering}

System frequency control will cause slow gradual changes in the measured signals, such characteristic is a signature of governing-control actions. Such deterministic components have to be removed and, since the frequency components related to these control actions are of lower frequency compared to electromechanical modes, they can be attenuated using a high pass filter. The mean of the filtered signal should be subtracted so that the signal fluctuates with reference to the same value at all times.

\subsection{Downsampling}

Sampling rates that are significantly faster than the power systems electromechanical dynamics will result in redundant data that will slow down computations and demand high processing power. Furthermore, when parametric methods are used, the autocovariance matrix that these methods use will be ill-conditioned for high sampling frequencies resulting in poor numerical sensitivity. Consider for example the conditioning of the Yule-Walker autocovariance matrix shown in Fig.3(b).

The amount of redundant data that can be safely removed without losing valuable information is given by the Nyquist-Shannon sampling theorem [5]. Since electromechanical modes are located in the frequency range up to $2 \mathrm{~Hz}$ [3], perfect reconstruction is guaranteed for sampling frequencies equal to or higher than $4 \mathrm{~Hz}$. To avoid errors due to aliasing the signal must be passed through a low pass filter prior to being downsampled. The filter should remove all frequency content above the Nyquist frequency [5] (half the sampling frequency).

\section{MODE FREQUENCY ESTIMATION}

By estimating the power spectral density (PSD) from a stochastic process, periodicities which are related to the dynamical behavior of a system may be detected. Modal frequencies will show up as peaks in the PSD, so if the PSD can be estimated, this will in turn give a estimate of mode frequencies. There exist many methods for PSD estimation; those utilized in this section and their methodology are described in [6].

\subsection{Parametric Spectral Estimation - Yule-Walker Spectral Estimator}

Assume that the PSD of a process can be completely described by $k$ parameters $a_{1}, a_{2}, . ., a_{k}$

$$
S(f)=S\left(f, a_{1}, a_{2}, . ., a_{k}\right)
$$


and that these parameters can be estimated from observations $X_{1}, X_{2}, \ldots, X_{N}$ of the system.

The PSD can then be estimated with

$$
\hat{S}(f)=S\left(f, \hat{a}_{1}, \hat{a}_{2}, . ., \hat{a}_{k}\right) .
$$

The Yule-Walker equations are used to estimate the parameters $\hat{a}_{1}, \hat{a}_{2}, \ldots, \hat{a}_{k}$. This Spectral Estimator utilizes the autoregressive model of order $p$

$$
Y_{t}=\phi_{1, p} Y_{t-1}+\phi_{2, p} Y_{t-2}+\ldots+\phi_{p, p} Y_{t-p}+\epsilon_{t}
$$

where $Y_{t}$ is a zero mean stationary process and $\phi_{1, p}, \phi_{2, p}$, $\ldots, \phi_{p, p}$ are the model coefficients. $\epsilon_{t}$ is a white noise process with zero mean and variance $\sigma_{p}^{2}$.

The PSD is then given by

$$
S(f)=\frac{\sigma_{p}^{2} \Delta t}{\left|1-\sum_{j=1}^{p} \phi_{j, p} e^{-i 2 \pi f_{j} \Delta t}\right|} .
$$

To obtain the model coefficients (3) is multiplied with $Y_{t-k}$, and then the expectation is obtained in order to determine the autocovariance sequence $s_{k}$. By using an available time series $X_{1}, X_{2}, . ., X_{N}$ estimates of $s_{k}$ can be produced and then be used to estimate the model coefficients.

$$
\hat{s}_{k}=\frac{1}{N} \sum_{t=1}^{N-|k|} X_{t} X_{t+|k|}, \hat{\phi}_{p}=\hat{\Gamma}_{p}^{-1} \hat{\gamma}_{p}
$$

$\sigma_{p}$ can be estimated by using the fact that $E\left[\epsilon Y_{t-k}\right]=0$ for $k>0$, and hence $\sigma_{p}^{2}$ can be estimated by $\hat{\sigma}_{p}^{2}=\hat{s}_{0}-$ $\sum_{j=1}^{p} \hat{\phi}_{j, p} \hat{s}_{j}$.

The complete PSD estimator can now be constructed as

$$
\hat{S}(f)=\frac{\hat{\sigma}_{p}^{2} \Delta t}{\left|1-\sum_{j=1}^{p} \hat{\phi}_{j, p} e^{-i 2 \pi f j \Delta t}\right|} .
$$

\subsection{Nonparametric Spectral Estimation}

The key concept in nonparametric spectral estimation is to use the following relation between the autocovariance sequence and the PSD

$$
S(f)=\Delta t \sum_{\tau=-\infty}^{\infty} s_{\tau} e^{-i 2 \pi f \tau \Delta t}
$$

and to consider a zero mean stationary time series of $N$ observations to estimate the autocovariance sequence for $\tau=0, \pm 1, \ldots, \pm(N-1)$.

$$
\hat{s}_{\tau}=\frac{1}{N} \sum_{t=1}^{N-|\tau|} X_{t} X_{t+|\tau|} .
$$

This estimate can be included into (7) to obtain the estimator:

$$
\hat{S}(f)=\frac{\Delta t}{N}\left|\sum_{t=1}^{N} X_{t} e^{-i 2 \pi f \tau \Delta t}\right|^{2} .
$$

This function is known as the periodogram. There are some major drawbacks with this type of estimators, the most important being high variance as well as severe bias caused by spectral leakage.

There are a few ways to modify the periodogram to get a estimator with better variance and bias properties.

\subsubsection{Welch's Spectral Estimator}

Welch's estimator is a modified version of the periodogram described in the previous section. The following steps are performed to form this estimator:

1. Split the original $\mathrm{N}$ observations into $N_{B}$ overlapping blocks with $N_{S}$ samples each.

2. Apply a data taper, $h_{t}$, on each block.

To reduce the bias due to spectral leakage it is recommended that every block is windowed with a Hanning data taper.

3. Obtain a periodogram for each block.

4. Average the individual periodograms together to form an overall spectral estimate.

The resulting spectral estimator will be given by

$$
\hat{S}(f)=\frac{\Delta t}{N_{B}}\left|\sum_{j=0}^{N_{B}-1} \sum_{t=1}^{N_{s}} h_{t} X_{t+j n} e^{-i 2 \pi f t \Delta t}\right|^{2}
$$

where $n$ is an integer specifying the amount of overlap between each block.

\subsubsection{Multitaper Spectral Estimator}

Suppose we have the time series $X_{1}, X_{2}, \ldots, X_{N}$ with zero mean and variance $\sigma^{2}$. For a sequence of $K$ data tapers, $h_{t, k}$, we can then compute $K$ individual eigenspectrums as follows:

$$
\hat{S}_{k}(f)=\Delta t\left|\sum_{t=1}^{N} h_{t, k} X_{t} e^{-i 2 \pi f t \Delta t}\right|^{2}
$$

The tapering will reduce bias in the estimator, and if the average of all these eigenspectrums are obtained, the variance of the resulting estimator will be reduced also. Therefore, the estimator will be given by

$$
\hat{S}(f)=\frac{1}{K} \sum_{k=0}^{K-1} \Delta t\left|\sum_{t=1}^{N} h_{t, k} X_{t} e^{-i 2 \pi f t \Delta t}\right|^{2}
$$

To get a good reduction in both bias and variance it is of great importance to select a sequence of data tapers that not only have good leakage properties, but also have relatively uncorrelated eigenspectrums. If the spectrums are too highly correlated, it will no be possible to get the desired reduction in variance. When data tapers are orthogonal, it is possible to obtain a sufficient degree of uncorrelation in a sequence of tapers, therefore: $\sum_{N}^{t=1} h_{t, j} h_{t, k}=0 \quad j \neq k$. The discrete prolate spheroidal sequences (dpss) fulfill these requirements and are therefore frequently used with multitaper spectral estimators.

\subsection{Parameter and block size selection}

For a sufficient amount of data, nonparametric spectral estimators (such as Welch) can be expected to produce high resolution PSDs with low variance and low bias. Consequently, the Welch Spectral Estimator will, for a sufficient amount of data, give a good overview of the 


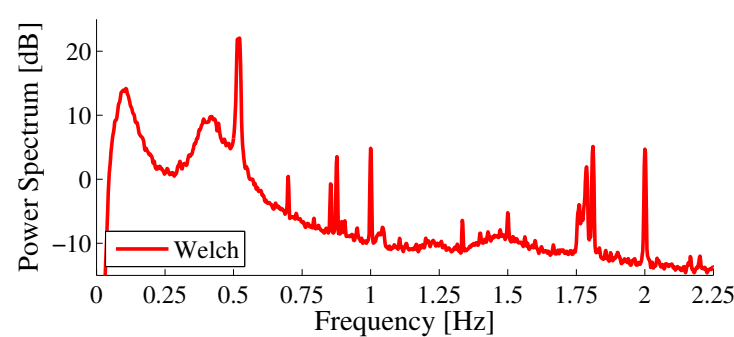

Figure 4. Welch Spectral Estimate, $12 \mathrm{~h}$ of data from

Tampere's PMU and 1440 samples block size.

location of the electromechanical modes in the system, as shown in Fig. 4.

This estimate can also be of great help when determining appropriate parameters, i.e. number of tapers, model order and block size for estimators using significantly less data.

A spectrogram shows how the spectral density of a signal varies with time, it can therefore be used to study variations and small fluctuations of modes in a power system. To get a spectrogram with reasonable resolution the individual PSDs must be computed with relatively few samples, in this case we use 10 minutes of data with a $90 \%$ data overlap between two successive PSDs.

To determine parameters for the different estimators, several spectrograms can be computed using different parameter values and then be compared to the 12-hour PSD. The spectrogram that is able to clearly capture the modes the most, will thus indicate the adequate parameter values to be used.

\subsubsection{Welch's Spectral Estimator - Block Size}

A decreased block size leads to a decrease in variance and lowered spectral resolution. By starting with a large block size and slowly decreasing it until the peaks can be distinguished from the variance, it is possible to obtain a PSD with acceptable variance while lowering the spectral resolution as little as possible. A $12 \mathrm{~h}$ Welch Spectrogram with the block size selected with this methodology is shown in Fig. $5^{3}$.

\subsubsection{Multitaper Spectral Estimator - Number of Tapers}

To determine an appropriate number of tapers we can use an approach similar to that used for the Welch spectrogram. Since the variance decreases as the number of tapers increases, we can begin with a small number of tapers and increase until the smaller peaks can be discerned from the variance. A $12 \mathrm{~h}$ Multitaper Spectrogram with the number of tapers selected with this methodology is shown in Fig.6.

\subsubsection{Yule-Walker Spectral Estimator - Model Order}

If the Yule-Walker model order is selected too high the spectrogram will be exhibiting artificial peaks; on the other hand, an insufficient model order results in a spectrogram where some of the true peaks of the modal frequencies have not been captured. By starting with a low model order and increasing it until all the peaks are visible an appropriate model order can be found. A 12h Yule-Walker Spectrogram

\footnotetext{
3 For Figs. 5-7, the measurement recording capture start time is 08 Aug-2010 00:00:00 UTC. All spectrograms are obtained using 12 hrs of data from Tampere's PMU and the PSDs are estimated using 10 min data parcels with $90 \%$ data overlap.
}

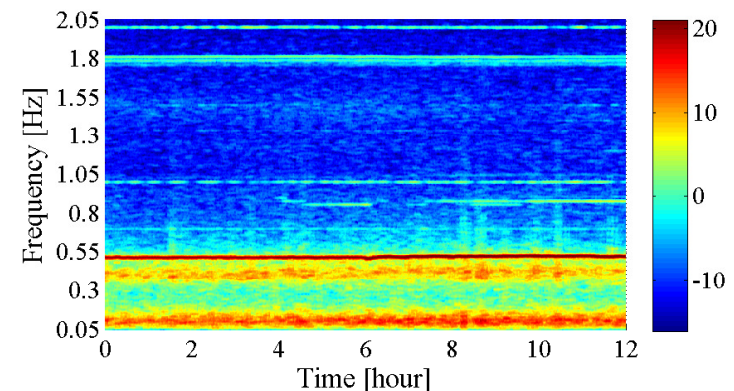

Figure 5. Welch Spectrogram, 500 samples block size.

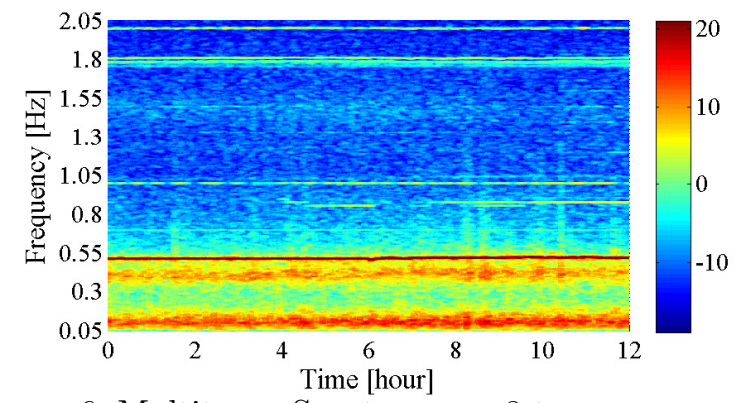

Figure 6. Multitaper Spectrogram, 8 tapers.

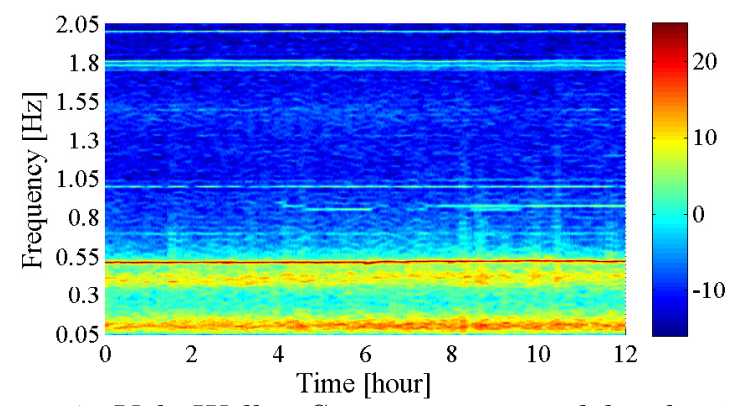

Figure 7. Yule-Walker Spectrogram, model order 160.

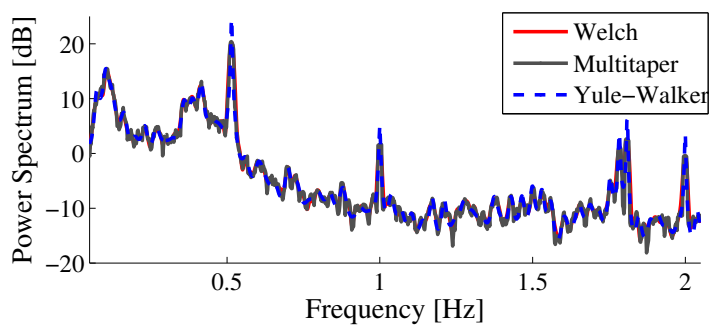

Figure 8. Spectral Estimates, 10min of data from Tampere.

with the model order selected with this methodology is shown in Fig.7.

\section{COMPARISON OF SPECTRAL ESTIMATORS}

Three different spectral estimators, Welch, Multitaper and Yule-Walker, have been used to compute spectrograms and PSD for the same data set. In Fig. 8 the PSDs are plotted together.

The main argument for using the Multitaper estimator over Welch is that there is less spectral leakage for spectra having very high dynamic ranges. However, we do not have very high dynamic ranges and so the estimators can be expected to have similar performance. As it can be seen the nonparametric estimators give very similar estimates, all the major peaks are more or less identical.

For the Yule-Walker estimator one can expect to get lower variance than for the nonparametric estimators. As it can 
Table 1. Mode frequencies from FFTs for data from Tampere's PMU.

\begin{tabular}{|l|l|}
\hline Mode Frequency $[\mathrm{Hz}]$ & Mode Type \\
\hline $0.3711-0.4395$ & Inter-area \\
\hline $0.5176-0.5273$ & Inter-area \\
\hline $0.6934-0.7324,0.8008-0.918,1.748-$ & Local \\
$1.758,1.776-1.789,1.807-1.816$ & \\
\hline
\end{tabular}

be seen from Fig.8 it is possible to obtain a PSD with lower variance, but the reduction is very small. This is because the system is complex, with many peaks, and some of them located closely together and so a high model order is required so that these modes are not compressed into one.

\subsection{Forced Oscillations}

There are forced oscillations (or undamped sinusoids) of unknown origin in the data. These oscillations will show up as narrow band oscillations in PSDs and spectrograms, and if superimposed over a real system mode, they will cause problems to obtain good damping estimates. FFTs (Fast Fourier Transforms) is used to examine the data and identify forced oscillations.

In our analysis forced oscillations appear in the data from all measurement locations. In common to all units are forced oscillations at exactly $1 \mathrm{~Hz}$ and $2 \mathrm{~Hz}$. It should be noted also that the amount of forced oscillations observed depends much on measurement location; units placed in the distribution grid (Tampere and LTU in Fig.9(b)) evince far more forced oscillations than the units placed in the transmission grid (Hasle in Fig.9(a)).

\subsection{Mode Frequency Estimates}

Forced oscillations have all spectral content concentrated in one small frequency bin while the modes spectral content is spread around the main peak. By inspecting FFTs we can separate forced oscillations from electromechanical modes and determine the mode locations; the modes shown in Tab. 1 could be observed in Tampere.

\section{MODE DAMPING ESTIMATION}

In the following section the performance of three methods for mode damping estimation have been tested, and their tolerance to forced oscillations is evaluated.

\subsection{The Autoregressive Yule-Walker Model}

The damping ratio of a mode can be extracted from the autoregressive Yule-Walker model. The model coefficients are the poles corresponding to the the pole polynomial coefficients of an input-output transfer function. The innovation variance corresponds to the numerator of this

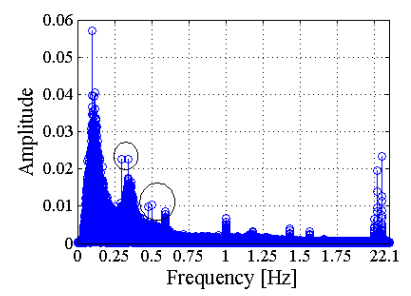

(a)

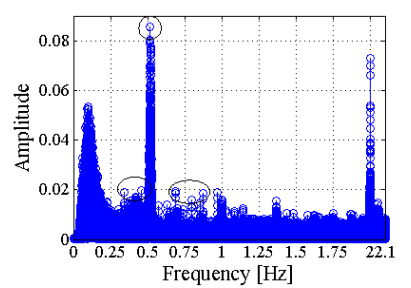

(b)
Figure 9. (a) FFT using $12 \mathrm{~h}$ of data from Hasle's PMU, (b) FFT using $12 \mathrm{~h}$ of data from LTU's PMU. transfer function, hence the input/output transfer function is given by

$$
H(s)=\frac{\sigma_{p}}{\sum_{i=1}^{p}\left(s-\phi_{i, p}\right)} .
$$

Using (3) the mode frequencies and corresponding damping ratios can be calculated from the poles (eigenvalues) of the transfer function as follows:

$$
\begin{gathered}
\lambda_{i}=\sigma_{i} \pm j \omega_{i}=\Re\left\{\phi_{i, p}\right\} \pm \Im\left\{\phi_{i, p}\right\} \\
f_{i}=\frac{\omega_{i}}{2 \pi}=\frac{\Im\left\{\phi_{i, p}\right\}}{2 \pi} \\
\zeta_{i}=\frac{-\sigma_{i}}{\sqrt{\sigma_{i}^{2}+\omega_{i}^{2}}}=\frac{-\Re\left\{\phi_{i, p}\right\}}{\sqrt{\Re\left\{\phi_{i, p}\right\}^{2}+\Im\left\{\phi_{i, p}\right\}^{2}}}
\end{gathered}
$$

\subsection{Half-Power Point Method}

By examining the peak width of a mode, damping can be approximated; a narrow peak means a low-damped mode while a wide peak means that the mode is well damped. The half-power point method states that the distance between the two half-power points surrounding the peak center is roughly proportional to the mode damping ${ }^{4}$.

The method is based on the assumption that the frequency response of the system can be modeled as:

$$
|H(\omega)|=\frac{1}{\sqrt{\left(1-\left(\frac{\omega}{\omega_{n}}\right)^{2}\right)^{2}+\left(2 \zeta \frac{\omega}{\omega_{n}}\right)^{2}}}
$$

A mode will thus appear at the frequency $\omega=\omega_{n}$ and the damping of this mode can be derived from the equation above yielding:

$$
\zeta=\frac{\omega_{2}-\omega_{1}}{2 \omega_{n}}
$$

where $\left|H\left(\omega_{1}\right)\right|=\left|H\left(\omega_{2}\right)\right|=\frac{1}{\sqrt{2}}\left|H\left(\omega_{n}\right)\right|$.

\subsection{Random Decrement Technique and Pronys Method}

There exist many methods for damping estimation from transient data; the Eigenvalue Realization Algorithm (ERA) and Prony's Method are often used for this. However, if a data set has more ambient characteristics and does not contain any transients of significant size, these methods will perform poorly. To overcome this, all the minor transients from the data can be collected and used to construct a transient of sufficient size for the methods to perform properly. This is the main concept behind the random decrement technique.

To detect and collect minor transients in the data, a threshold is set and every time the signal exceeds this threshold the data sequence is collected. The collected data sequences will then be averaged to create a transient:

$$
y(k)=\sum_{k=1}^{n} \frac{1}{N} \sum_{i=1}^{N} y_{i, k}
$$

where $y$ is the data sequence, $N$ is the number of collected sequences and $\mathrm{n}$ is the number of samples in each sequence. With the transient $y(k)$, Prony's Method can be used to

4 PSDs from the Welch spectral estimator are used to compute the half-power point damping estimates in this Section. 
Table 2. Mode frequency, damping and variance from the methods.

\begin{tabular}{|l|l|l|l|}
\hline Method & $\bar{f}(\mathrm{~Hz})$ & $\bar{\zeta}(\%)$ & Variance \\
\hline Yule-Walker & 0.8342 & 6.5826 & 0.3564 \\
\hline Half-Power Point & 0.8324 & 6.7714 & 0.4215 \\
\hline Random Decrement & 0.8312 & 7.0311 & 0.5095 \\
\hline
\end{tabular}

extract the mode frequencies and damping. This method is designed to fit a transient to the function

$$
\hat{y}(t)=\sum_{i=1}^{P} R_{i} e^{\lambda_{i} t}
$$

where $R_{i}$ is the residue associated with mode $\lambda_{i}$. These parameters can be obtained by fitting, in a least square sense, $\hat{y}(t)$ to the system output $y(k)$ and then the damping can be calculated with (14).

\subsection{Simulations}

To verify that the damping estimation algorithms in this section perform correctly, simulations are carried out. A model of the Southern/Southeastern Brazilian power system [7], with one inter-area mode of $0.8328 \mathrm{~Hz}$ and $6.93 \%$ damping, is used for this purpose.

By using white noise and small steps as inputs to all load buses, ambient data containing small transients was generated. For 12 hours of simulated data the methods give, using 10 minute blocks, the estimates shown in Tab. 2 . All three methods give comparable results, with high correlation of the estimates. The most significant difference is that the random decrement has slightly higher variance than the other two methods.

\subsection{Effect of Forced Oscillations and Undamped Sinusoids}

Forced oscillations will, if superimposed over a system mode, have negative effects when computing accurate damping estimates. By computing the Welch spectral estimate before and after an undamped sinusoid with the same frequency as the added mode, it can be seen how the performance of the half-power point method is affected by the undamped sinusoid.

From Fig.10, it can be seen that an undamped sinusoid will have a huge impact on the damping estimates obtained. The half-power points will be very close together and equation (18) will give a much too small damping ratios.

For the Yule-Walker AR model, the undamped sinusoid will decrease the real part of the eigenvalues, while the imaginary part will be relatively unaffected, and so equation (16) will also yield much too small damping ratios.

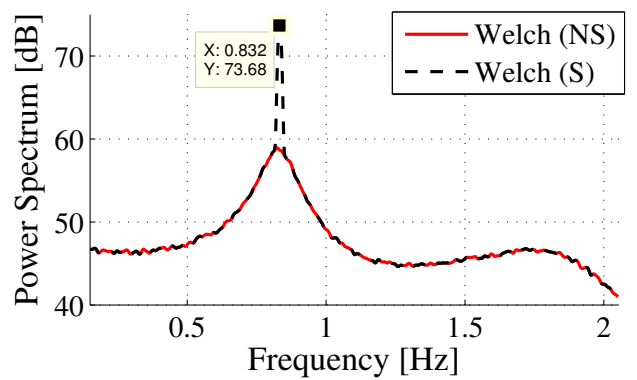

Figure 10. Welch spectral estimates with superimposed forced oscillation (S) and without (NS).
The random decrement method on the other hand is much more tolerant to forced oscillations; a small oscillation will only cause a small decrease in damping. This is because the collected transients are in general of much larger amplitude than the forced oscillations and the decrease in damping is proportional to the ratio between these two.

\section{CONCLUSIONS AND DISCUSSION}

This article has shown how different spectral analysis techniques can be applied to synchrophasor measurements originating from the Nordic grid to estimate low-frequency electromechanical oscillations.

A clear and systematic approach for data preprocessing catering to the unique imperfections of PMU data has been presented and illustrated; for each step in this process we provide a well-founded rationale.

For mode frequency estimation, the article provides a practical methodology for selecting the parameters required by parametric estimators and non-parametric estimators (MTM), as well as the block sizes used by non-parametric estimators. A comparison of spectral estimation results shows the applicability of this approach. Moreover, this article shows the first application of the MTM method in the literature.

Analysis of PSDs obtained from different estimators revealed the presence of narrow frequency band components. Through FFT analysis of data both from low-voltage as well as high voltage PMU installations, the existence of these components was confirmed.

For mode damping estimation, three algorithms capable of providing damping estimates under ambient data were tested. The performance of these estimators was evaluated through simulations. The simulation results suggest that the random decrement algorithm has a more acceptable performance under the presence of forced oscillations. This hypothesis is currently being scrutinized by the authors' for the case of real PMU data.

The most important realization of this article is that to improve the quality of mode estimates and in order to be able to obtain reliable damping estimates, methods that can detect and remove the effects of forced oscillations in the measurements need to be developed.

\section{REFERENCES}

[1] K. Uhlen, et al, "Application of linear analysis for stability improvements in the Nordic power transmission system," IEEE Power Engineering Society General Meeting, 2003, vol.4, pp. 4, 13-17 July 2003.

[2] J. Hauer, et al, "Integrated Dynamic Information for the Western Power System: WAMS Analysis in 2005," Chapter 14 in Power System Stability and Control: Electric Power Engineering Handbook, pp. 14-1 - 14-51, CRC Press: Boca Raton, FL, 2007.

[3] D. Trudnowski and J. W. Pierre, "Signal processing methods for estimating small-signal dynamic properties from measured responses," in Inter-area Oscillations in Power Systems: A Nonlinear and Nonstationary Perspective. Springer: New York, NY, 2009; pp. 1-36.

[4] L. Vanfretti, et al, "Application of Ambient Analysis Techniques for the Estimation of Electromechanical Oscillations from Measured PMU Data in Four Different Power Systems", European Transactions on Electrical Power, vol. 21, no. 4, pp. 1640-1656, 2011.

[5] Torkel Glad and Lennart Ljung, "Control Theory - Multivariate and Nonlinear Methods", Taylor \& Francis 2000

[6] David B. Percival and Andrew T. Walden, "Spectral Analysis for Physical Applications - Multitaper and Conventional Univariate Techniques", Cambridge University Press 1998.

[7] N. Martins and L. T. G. Lima. "Eigenvalue and frequency domain analysis of small-signal electromechanical stability problems." In IEEE/PES Symposium on Applications of Eigenanalysis and Frequency Domain Methods, 1989. 